\title{
VECTOR INDICATOR AS A TOOL OF RECURRENT ARTIFICIAL NEURON NET FOR PROCESSING DATA
}

\author{
Alexander Trunov \\ First Vice-Principal \\ Department of Automatization and Computer Integrated Technologies \\ Petro Mohyla Black Sea National University \\ 68 Marines str., 10, Nikolaev, Ukraine, 54003 \\ trunovalexandr@gmail.com
}

\begin{abstract}
The three-level comparator is applied as a tool to formation of vector-indicator of function and its argument and recurrent artificial neuron net (RANN). The modernization of expression in Taylor series due to usage of the vector-indicator components is introduced. The example of RANN for peripheral processing data on the basis of a long-short-term memory is proposed. The dependence of number point shift from order of oldest derivatives in expression is studied. The system equations realizing conditions of minimization a sum of squared deviations from the patterns are written. The processes of transformation on different stage of data acquisition and processing into RANN are considered. Decomposition of function on derivatives and vector-indicator inside RANN is shown. The numerical experiments for analytical learning are done, they demonstrated convergence of analytical learning algorithms independently from first approximation even for oscillating operators.
\end{abstract}

Keywords: three-level comparator, vector-indicator, RANN, analytical learning, convergence.

\section{Introduction}

Demonstration of advantages on application of complex approach [1-9] to design data acquisition, initial processing and its collecting for search of mathematical models, to creation and processing data base and base of knowledge as intellectual activity process that in its own majority realizes due to comparative analysis [2]. Its application to the analysis in the problems of prediction, prevention and fault removal allows to create algorithms and schemes for development of these type devices [10]. Further development of comparative approach leads to the necessity of observation of qualitative features of behavior of a physical quantity for variation of integer-valued quantities [3,11]. The last play the role of indicators and can be applied to formation of productive rulers and regulations. Development of the initial data processing [6], which recently implemented in automated systems [7], in the production [8], which flexibly rebuilt and observation system [9], leads to the necessity of further development of rapid methods of qualitative analysis and methods of formation based on the results of conclusions [10].

The main unresolved problem is searching a means of submitting decomposition of dynamic signal through the new instrument of vector-indicators and by recurrent network, based on longshort-term memory.

The aim of this work is to form a recurrent network for initial processing of sensors information based on instrument of vectors-indicators and recurrent approximation.

\section{Materials and Methods}

In articles [1-4] are determined the different classes of data to be processed. Considering the generalized information flow based on assumptions about the existence and continuity, in the work are used methods functional analysis, comparative methodology [2, 3] of intellectual activity, methods of forming neural networks and RANN, recurrent approximation method for solving the problem of analytic training neurons [10-12].

\section{Experimental procedures}

\section{1. Taylor expansion in series of scalar function by using vectors-indicators}

Let us introduce on the unlimited and ordered set $\forall \mathrm{Y} \in(-\infty, \infty)$ the rules for actuation a trigger that is defined by three $\mathrm{Y}_{1}, \mathrm{Y}_{2}, \mathrm{Y}_{3}$-standards such as: 


$$
\mathrm{D}_{3}(\mathrm{Y})=\left\{\begin{array}{lll}
-1, & \text { if } & \mathrm{Y}<\mathrm{Y}_{1} \\
0, & \text { if } & \mathrm{Y}=\mathrm{Y}_{2} \\
1, & \text { if } & \mathrm{Y}>\mathrm{Y}_{3}
\end{array}\right.
$$

Let notice, that entered the operator $\mathrm{D}_{3}$, that acts on scalar-function and makes image in form of scalar that is displayed in the metric space. Under these conditions and signs, expansion of arbitrary scalar-function of a vector argument will be submitted:

$$
\begin{aligned}
\mathrm{L}_{\mathrm{i}}\left(\overline{\mathrm{x}}_{\mathrm{p}}+\Delta \overline{\mathrm{x}}_{\mathrm{p}}\right) & =\left|\mathrm{L}_{\mathrm{i}}\left(\overline{\mathrm{x}}_{\mathrm{p}}\right)\right| \mathrm{D}_{3}\left(\mathrm{~L}_{\mathrm{i}}\left(\overline{\mathrm{x}}_{\mathrm{p}}\right)\right)+\sum_{\mathrm{j}=1}^{\mathrm{n}}\left|\frac{\partial \mathrm{L}_{\mathrm{i}}\left(\overline{\mathrm{x}}_{\mathrm{p}}\right)}{\partial \mathrm{x}_{\mathrm{j}}}\right| \mathrm{D}_{3}\left(\frac{\partial \mathrm{L}_{\mathrm{i}}\left(\overline{\mathrm{x}}_{\mathrm{p}}\right)}{\partial \mathrm{x}_{\mathrm{j}}}\right) \Delta_{\mathrm{X}_{\mathrm{j}}}+ \\
+ & \sum_{\mathrm{k}=1}^{\mathrm{n}} \sum_{\mathrm{j}=1}^{\mathrm{n}}\left|\frac{\partial^{2} \mathrm{~L}_{\mathrm{i}}\left(\overline{\mathrm{x}}_{\mathrm{p}}\right) \mid}{\partial \mathrm{x}_{\mathrm{k}} \partial \mathrm{x}_{\mathrm{j}}}\right| \mathrm{D}_{3}\left(\frac{\partial^{2} \mathrm{~L}_{\mathrm{i}}\left(\overline{\mathrm{x}}_{\mathrm{p}}\right)}{\partial \mathrm{x}_{\mathrm{k}} \partial \mathrm{x}_{\mathrm{j}}}\right) \frac{\Delta_{\mathrm{X}_{\mathrm{k}}} \Delta_{\mathrm{x}_{\mathrm{j}}}}{2} .
\end{aligned}
$$

This expansion is implemented under conditions existence of Fréchet derivatives from the first to the third order, and its accuracy is determined according to the theorem of the mean [5] by the maximum value of the derivative module of third order.

\section{2. Application of long-short-term memory to design of RANN}

Under these conditions, the use of recurrent networks with memory and structural elements that determine the components of the vector-indicator also requires an effective tool for analytical determination of roots. In the Fig. 1 are shown fragment of such RANN that generalizing the idea of a new form of Taylor decomposition and its practical application and the implementation of recurrent parallel signal processing. Thus, if the output of the neuron 3 defines standard for behavior of systems for any vector of strategies $\bar{X}$, and from neurons 4 and $17 \bar{X}_{n}$ and $\bar{X}_{n+1}$ then deviation:

$$
\Delta \mathrm{L}(\overline{\mathrm{X}})=\mathrm{L}(\overline{\mathrm{X}})-\mathrm{L}\left(\overline{\mathrm{X}}_{\mathrm{n}+1}\right),
$$

determines the strategy of control actions in accordance with changes the values of components of the vector-indicator, which is obtained after processing by comparator (1) of component vector (3). Furthermore, after adding to the output of the neuron 7 product output neuron 11 multiplied by the output of neuron 16, and add the product transposed vector of output from neuron 16 on output of neuron 15 and multiplied by half the vector output of neuron 16 get approximated value function in point $\overline{\mathrm{X}}_{\mathrm{n}+1}$ :

$$
\mathrm{L}\left(\overline{\mathrm{X}}_{\mathrm{n}+1}\right)=\left\|\mathrm{a}_{\mathrm{i}}\right\|+\left\|\mathrm{b}_{\mathrm{i}}\right\| \Delta \overline{\mathrm{X}}_{\mathrm{n}}+\Delta \overline{\mathrm{X}}_{\mathrm{n}}^{\mathrm{T}}\left\|\mathrm{c}_{\mathrm{ij}}\right\| \frac{\Delta \overline{\mathrm{X}}_{\mathrm{n}}}{2} .
$$

In turn, this standard element (Fig. 1) also makes it possible to estimate the approximation error by comparing the values $\mathrm{L}\left(\overline{\mathrm{X}}_{\mathrm{n}+1}\right)$ calculated by equation (4) and exit 17 . The latter, in turn, opens the possibility to carry out improvement model and formation of control rules. This is especially true for real systems in which $\mathrm{L}\left(\overline{\mathrm{X}}_{\mathrm{n}+1}\right)$ are oscillating nonsmooth functions, but it requires a lot of point approximations.

The number of points for information delays. Assume that on the input 1 acted first value $\mathrm{X}_{1}$, then after processing the output value will be created $\mathrm{L}\left(\mathrm{X}_{1}\right)$. It should be noted that only after acceptance on input 1 value $\mathrm{X}_{2}$ will be received new value $\mathrm{L}\left(\mathrm{X}_{2}\right)$ and new value of first derivative $\mathrm{L}^{\prime}\left(\mathrm{X}_{1}\right)$ will be calculated. By deduction is easy to verify, using one stepper memory the number of points required to calculation the derivative is on one more than the original order. Thus, after missing four points will be accumulated information about the behavior of the three derivatives in the first point, as the rate of change of derivatives and required accuracy requirements determined by speed of the network operation. The accumulation of such comprehensive information about the behavior of the object for distance of changing interval argument will build a model and assess its adequacy by one of the known methods.

Introduction of indicator for argument change. Most tasks of function research is a priori provided that the argument is changing in the direction of its growth. In the experimental study of 
the argument as a vector may vary in different directions, or to remain unchanged as a vector, or any of its components is to remain unchanged. Applying to the growth of argument comparator that implements the predicate for example in the form of (1) gets tool for analysis that complements toolkit vector-indicator (2) and (4) that is used to expansion in series vector-function by using vectorsindicators [4-9].

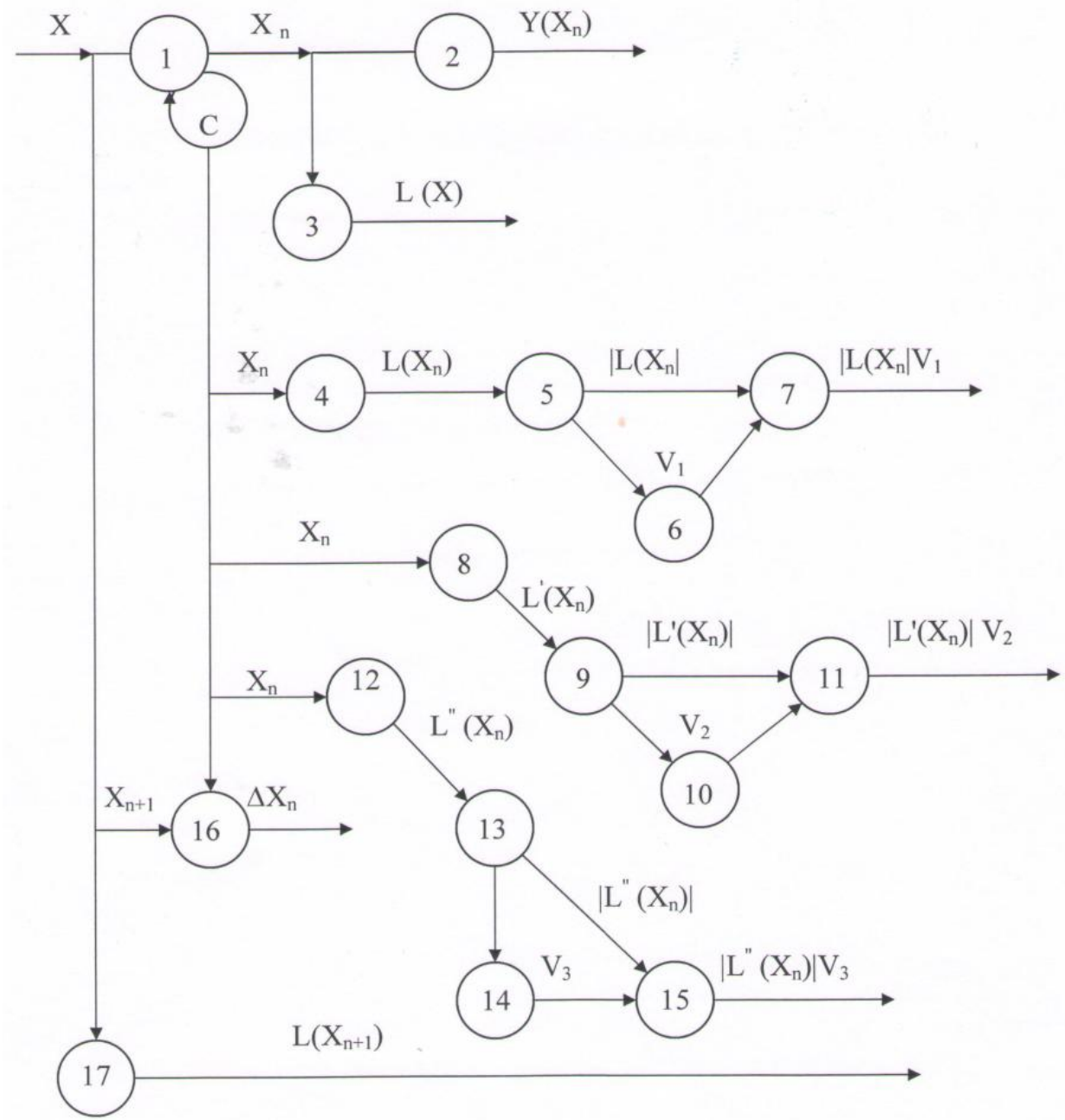

Fig. 1. The fragment of RANN long-short-term memory

Implementing simplified definition of growth in recurrent net. So giving in a one moment of time vector argument from the entrance of the network and the inner layer of neuron 1-2, which are always shifted on one step, to the neuron 16, growth of argument is observed. Applying to output signal from neuron 16 comparator, which implements the predicate in the form of (1) we obtain the output neuron 16 after processing as value of indicator:

$$
\mathrm{V}_{4}=\mathrm{D}_{3}(\Delta \overline{\mathrm{X}})
$$

\section{The practical definition of convergence for analytic training neurons}

Analytical training and retraining neurons was proposed and realized in [6] for normal neural network. It should be noted that the proposed in [6] recurrent solutions under conditions of minimizing the sum of squared errors, as fragment RANN, reduced to solving systems of nonlinear equations. Confine ourselves to the case of searching for synaptic weights coefficients of the $\mathrm{j}$-th neuron to the $\mathrm{K}$ standards and writes key's system algorithm: 


$$
\left\{\begin{array}{l}
\sum_{\mathrm{k}=1}^{\mathrm{K}}\left(\mathrm{Y}_{\mathrm{i}}-\frac{1}{1+\mathrm{e}^{-\mathrm{S}_{\mathrm{i}}}}\right) \frac{\mathrm{e}^{-\mathrm{S}_{\mathrm{i}}}}{\left(1+\mathrm{e}^{-\mathrm{S}_{\mathrm{i}}}\right)^{2}}=0 ; \mathrm{k}=\overline{1, \mathrm{~K} ; \mathrm{i}}=\overline{1, \mathrm{q}}, \\
\sum_{\mathrm{k}=1}^{\mathrm{K}}\left(\mathrm{Y}_{\mathrm{i}}-\frac{1}{1+\mathrm{e}^{-\mathrm{S}_{\mathrm{i}}}}\right) \frac{\mathrm{X}_{\mathrm{kie}}{ }^{-\mathrm{S}_{\mathrm{i}}}}{\left(1+\mathrm{e}^{-\mathrm{S}_{\mathrm{i}}}\right)^{2}}=0 ; \mathrm{S}_{\mathrm{i}}=\sum_{\mathrm{j}=1}^{\mathrm{q}}\left(\omega_{0 \mathrm{i}}+\mathrm{x}_{\mathrm{ki}} \omega_{\mathrm{ji}}\right) .
\end{array}\right.
$$

Table 1

Value of synaptic weights coefficients for recurrent network and n-th approach

\begin{tabular}{ccccc}
\hline $\mathbf{n}$ & $\boldsymbol{\omega}_{\mathbf{0}}$ & $\boldsymbol{\omega}_{\mathbf{1}}$ & $\boldsymbol{\omega}_{\mathbf{0}}$ & $\boldsymbol{\omega}_{\mathbf{1}}$ \\
\hline 0 & -0.5 & 0.5 & -1 & 1 \\
1 & 0.348296 & 1.918929 & 0.226463 & 2.736287 \\
2 & 0.048179 & 2.869919 & -0.09139 & 3.693219 \\
3 & -0.18609 & 3.853674 & -0.30202 & 4.651896 \\
4 & -0.34588 & 4.794877 & -0.40256 & 5.488551 \\
5 & -0.41371 & 5.590638 & -0.42399 & 6.105785 \\
6 & -0.42476 & 6.170496 & -0.4239 & 6.473803 \\
7 & -0.42376 & 6.503514 & -0.42319 & 6.603236 \\
8 & -0.42376 & 6.503514 & -0.42311 & 6.617249 \\
9 & -0.42316 & 6.608308 & -0.42311 & 6.6174 \\
10 & -0.42316 & 6.608308 & -0.42311 & 6.6174 \\
11 & -0.42311 & 6.617338 & -0.42311 & 6.6174
\end{tabular}

Further analysis (5) demonstrated principle and practical applicability and its applicability to the analytical study of recurrent networks. However, oscillations of operators significantly worsened convergence, therefore was used numerical experiment for the study of the nature of convergence. As indicate results of data analysis into Table 1 values of synaptic weight coefficients, independently from the values selected for the initial approach, converge to the same values with accuracy up to a fifth sign. Artificial change of the initial approximation also demonstrates the presence of convergence and algorithm stability to choosing the initial approximations that confirming the results of the calculations presented into Table 1-3. The first and second column of Table 1 are present data that was calculated without vector-indicator, but the other with its use and the using of productive rules [11].

Table 2

Value of synaptic weights coefficients for recurrent network and n-th approach

\begin{tabular}{ccccc}
\hline $\mathbf{n}$ & $\boldsymbol{\omega}_{\mathbf{0}}$ & $\boldsymbol{\omega}_{\mathbf{1}}$ & $\boldsymbol{\omega}_{\mathbf{0}}$ & $\boldsymbol{\omega}_{\mathbf{1}}$ \\
\hline 0 & 0.5 & 0.5 & 1 & 1 \\
1 & 0.243522 & 1.506316 & 0.226463 & 2.736287 \\
2 & 0.051021 & 2.453176 & -0.09139 & 3.693219 \\
3 & -0.15255 & 3.439809 & -0.30202 & 4.651896 \\
4 & -0.31466 & 4.403316 & -0.40256 & 5.488551 \\
5 & -0.40187 & 5.262655 & -0.42399 & 6.105785 \\
6 & -0.42418 & 5.937241 & -0.4239 & 6.473803 \\
7 & -0.4244 & 6.384425 & -0.42319 & 6.603236 \\
8 & -0.42331 & 6.582194 & -0.42311 & 6.617249 \\
9 & -0.42312 & 6.616483 & -0.42311 & 6.6174 \\
10 & -0.42311 & 6.617399 & -0.42311 & 6.6174 \\
11 & -0.42311 & 6.6174 & -0.42311 & 6.6174 \\
12 & -0.42311 & 6.6174 & -0.42311 & 6.6174 \\
13 & -0.42311 & 6.6174 & -0.42311 & 6.6174
\end{tabular}


Table 3

Value of synaptic weights coefficients for recurrent network and n-th approach

\begin{tabular}{ccccc}
\hline $\mathbf{n}$ & $\boldsymbol{\omega}_{\mathbf{0}}$ & $\boldsymbol{\omega}_{\mathbf{1}}$ & $\boldsymbol{\omega}_{\mathbf{0}}$ & $\boldsymbol{\omega}_{\mathbf{1}}$ \\
\hline 0 & 0.5 & 5 & -0.5 & 5 \\
1 & -0.6098 & 4.273501 & -0.43524 & 5.641739 \\
2 & -0.45191 & 4.896313 & -0.4261 & 6.190138 \\
3 & -0.43134 & 5.601353 & -0.42375 & 6.511489 \\
4 & -0.42602 & 6.165245 & -0.42316 & 6.609489 \\
5 & -0.42381 & 6.500538 & -0.42311 & 6.617353 \\
6 & -0.42317 & 6.60784 & -0.42311 & 6.6174 \\
7 & -0.42311 & 6.617331 & -0.42311 & 6.6174 \\
8 & -0.42311 & 6.6174 & -0.42311 & 6.6174 \\
9 & -0.42311 & 6.6174 & -0.42311 & 6.6174 \\
10 & -0.42311 & 6.6174 & -0.42311 & 6.6174 \\
11 & -0.42311 & 6.6174 & -0.42311 & 6.6174 \\
12 & -0.42311 & 6.6174 & -0.42311 & 6.6174
\end{tabular}

The changes sign of the initial approximation virtually doesn't impact on the availability of convergence and its character. The comparison of the values of the first and initial approximations of various characters and sign shows bilateral convergence. The nature of this convergence and the benefits of recurrent approximation method [10] show the data from the first and third column of Table 1. Thus, despite the oscillations - change the sign on the opposite, which observed in the first and second approximation, values of coefficients are coincided. However, after the first oscillation behavior of synaptic weights coefficients begins monotonously converges to the same values.

\section{Discussion of simulation results}

In the analytical study algorithms were used, for which training is based on the requirement of zero error value and minimize the sum of squared errors. Despite on their simplicity selection of predefined standards for training is limited by the presence of asymptotic points, both for the first and for the second approach. The latter is visualized particularly in the case of reducing the number of decomposition terms of operators to two. Application of vector-indicators improves the process of convergence, but increases the total amount of calculations. Development of the initial data processing leads to the necessity of further development of rapid methods for qualitative analysis and formation methods based on their results - conclusions [10]. Submission decomposition of dynamic signal through the new indicators (1), with recurrent network, based on long-short-term memory, simplifies the process of decomposition by paralleling analog processing or calculations. However, the introduction of the analysis values of these indicators, from point to point and forming productive rules for different types of tasks, creates new tools, for cybernetic methods of control. Certainly, the simplicity and the ability of these modules to embed in other systems and networks will be the main benefits. Nevertheless it should be noted: method is deprived of generality due to its complexity and for linear problems it is not justified. Benefits of these methods appear to nonlinear analysis, with oscillation processes or processes with pronounced of hysteresis. Comprehensive study fundamental properties of decomposition and behavior, both of the indicators and their changes obviously open new properties and possibilities of implementation as comparative approaches and such the new tools for vector-indicator and recurrent neural networks based on recurrent approximation.

\section{Conclusion}

1. Introduction of behavior physical quantity indicators and its first and second derivatives allows to decompose of continuous, differentiable operators and build a recurrent network for the initial data processing.

2. Application of vector-indicators makes RANN analytical learning algorithms practically independent from the choice of initial approximation. 


\section{References}

[1] Bodyanskiy, Ye., Chaplanov, O., Popov, S. (2003). Adaptive prediction of quasiharmonic sequences using feedforward network. Proc. Int. Conf. Artificial Neural Networks and Neural Information Processing, Istanbul, ICANN / ICONIP, 378-381.

[2] Kryuchkovskiy, V. V., Petrov, K. E. (2011). Development of methodology for identification models of intellectual activity. Problem of information technology, 9, 26-33.

[3] Kryuchkovskiy, V. V., Petrov, E. G. Brynza, N. A. (2010). Informativnaya predpochtitelnost statisticheskoy formy predstavleniya iskhodnykh dannykh $\mathrm{v}$ usloviyakh intervalnoy neopredelennosti. St. Petersburg state polytechnical university journal "Computer science. Telecommunications and control systems”, №4 (103), 11-18.

[4] Khodakov, V. E., Vezumskiy, A. K. (2013). Kharakternye osobennosti odnogo klassa sotsial'noekonomicheskikh sistem. Problemi Informatsiynikh, №2(014), 10-14

[5] Collatz, L. (1964). Funktionalanalisis und Numerische Mathematik. Springer-Verlag BerlinGottigen-Heidelberg, 447.doi: 10.1007/978-3-642-95028-5

[6] Kondratenko, Y. P., Sidenko, Ie. V. (2012). Correction of the Knowledge Database of Fuzzy Decision Support System with Variable Structure of the Input Data. Modeling and Simulation. Anna M. Gil-Lafuente, V.Krasnoproshin (Eds.). Proc. of the Int. Conference MS'12, (2-4 May 2012, Minsk, Belarus). - Minsk: Publ. Center of BSU, 56-61.

[7] Kondratenko, Y. P., Korobko, V. V. Korobko, O. V. (2013). Distributed computer system for monitoring and control of thermoacoustic processes. In: Intelligent Data Acquisition and Advanced Computing Systems IEEE 7th Int. Conf. on. IEEE, 249-253.doi: 10.1109/idaacs.2013.6662682

[8] Kondratenko, Y., Klymenko, L., Kondratenko, V., Kondratenko, G., Shvets E. (2013). Slip Displacement Sensors for Intelligent Robots: Solutions and Models. In: Intelligent Data Acquisition and Advanced Computing Systems IEEE 7th Int.onf. on. IEEE, 861-866.doi: 10.1109/idaacs.2013.6663050

[9] Fiesler, E., Duong, T., Trunov, A. (2000). Design of neural network-based microship for color segmentation. IEEE Transaction Intelligent Optical Systems, Pr. Of SPIE 4055, 228-238.

[10] Trunov, A. N. (2011). "Recurrence approximation in problems of modeling and design": Monografy - Mykolayiv: Petro Mohyla BSSU, 272.

[11] Trunov, A. N. (2013). Intellectualization of the models' transformation process to the recurrent sequence. European Applied Sciences, Ort Publishing, 9, 1, 123-130.

[12] Trunov, A. N. (2014). Application of the recurrent approximation method to synthesis of neuron net for determination the hydrodynamic characteristics of underwater vehicles. Problem of Information Technology, Jornal, 02(016), 39-47. 